\title{
A retrospective analysis on the utility and complications of upper arm ports in 433 cases at a single institute
}

\author{
Yukiko Mori $^{1} \cdot$ Satoshi Nagayama $^{2} \cdot$ Jun-ichiro Kawamura $^{2} \cdot$ Suguru Hasegawa $^{2}$. \\ Eiji Tanaka $^{2} \cdot$ Hiroshi Okabe $^{2} \cdot$ Megumi Takeuchi $^{3} \cdot$ Makoto Sonobe $^{4}$. \\ Shigemi Matsumoto ${ }^{1} \cdot$ Masashi Kanai $^{1} \cdot$ Manabu Muto $^{1} \cdot$ Tsutomu Chiba $^{1,5}$. \\ Yoshiharu Sakai ${ }^{2}$
}

Received: 13 July 2015 / Accepted: 9 October 2015 / Published online: 27 October 2015

(C) The Author(s) 2015. This article is published with open access at Springerlink.com

\begin{abstract}
Background We have employed upper arm central venous ports (UACVPs) since 2006 for long-term intravenous chemotherapy (CTx) or fluid supplementation. We evaluated the long-term availability of CVPs implanted in the upper arm to determine whether UACVPs could be one of the treatment options besides chest CVPs in terms of CVPrelated complications.

Methods We reviewed the medical records of all patients who underwent subcutaneous implantation of UACVPs at Kyoto University Hospital from 1 April, 2006 to 30 June, 2009. We assessed the indwelling duration of the UACVPs and the incidences of early and late UACVP-related complications.

Results A total of 433 patients underwent subcutaneous implantation of UACVPs during this time period.
\end{abstract}

Y. Mori and S. Nagayama contributed equally to this paper.

Satoshi Nagayama

nagayama@kuhp.kyoto-u.ac.jp

1 Department of Clinical Oncology, Kyoto University Hospital Cancer Center, 54 Shogoin Kawahara-cho, Sakyo-ku, Kyoto 606-8507, Japan

2 Division of Gastrointestinal Surgery, Department of Surgery, Kyoto University Hospital, 54 Shogoin Kawahara-cho, Sakyo-ku, Kyoto 606-8507, Japan

3 Department of Breast Surgery, Kyoto University Hospital, 54 Shogoin Kawahara-cho, Sakyo-ku, Kyoto 606-8507, Japan

4 Department of Thoracic Surgery, Kyoto University Graduate School of Medicine, Kyoto University, Yoshidahonmachi, Sakyo Ward, Kyoto, Kyoto 606-8501, Japan

5 Department of Gastroenterology and Hepatology, Kyoto University Hospital, 54 Shogoin Kawahara-cho, Sakyo-ku, Kyoto 606-8507, Japan
The cumulative follow-up period was 251,538 catheter days, and the median duration of UACVP indwelling was 439.0 days $(1-2,24)$. There was no UACVP-related mortality throughout the study period. A total of 83 UACVPrelated complications occurred (19.2\%), including 43 cases of infection (9.9\%, 0.17/1000 catheter days), ten cases of catheter-related thrombosis $(2.3 \%, 0.040 / 1000$ catheter days), ten cases of occlusion $(2.3 \%, 0.040 / 1000$ catheter days), nine cases of catheter dislocation $(2.0 \%$, $0.036 / 1000$ catheter days), five cases of port leakage (1.2\%, 0.019/1000 catheter days), four cases of skin dehiscence $(0.9 \%, 0.015 / 1000$ catheter days $)$ and two cases of port chamber twist $(0.5 \%, 0.008 / 1000$ catheter days). The removal-free one-year port availability was estimated at $87.8 \%$.

Conclusions UACVPs were of long-term utility, with complication rates comparable to those of chest CVPs previously reported.

Keywords Upper arm central venous ports · Long-term availability $\cdot$ CVP-related complications

\section{Introduction}

Central venous ports (CVPs) are good medical devices to facilitate the long-term administration of intravenous chemotherapy (CTx) or fluid supplementation. Since continuous systemic chemotherapy such as FOLFOX or FOLFIRI in combination with molecular-targeted drugs has been regarded as the standard treatment for advanced colorectal cancers [1-3], the subcutaneous implantation of CVPs has become an essential device in daily medical care. Moreover, since the combination regimen of 5-fluorouracil, folinic acid, oxaliplatin and irinotecan (FOLFIRINOX) has 
been proposed as a new standard of care for metastatic pancreatic cancer patients [4], the subcutaneous implantation of CVPs has been performed more frequently than ever before.

The most common site for the implantation of CVPs is the anterior chest via the subclavian vein (chest CVPs). However, the insertion of CVPs into the subclavian vein is sometimes complicated by pneumothorax, pneumohemothorax, or arterial punctures [5-8]. In addition, the longterm usage of chest CVPs is sometimes complicated with pinch-off syndrome, a severe complication in which the catheter becomes kinked, compressed or even fragmented at the narrow space between the clavicle and the first rib due to repetitive arm motion [9-11]. The implantation of CVPs via the internal jugular vein is considered to be safer compared to access via the subclavian vein [12-14]. In contrast, some authors prefer to implant CVPs in the upper arm or forearm via the basilic or axillary veins because of safer puncture procedures, and concluded that arm CVPs could be suitable for long-term usage with minimal complications [15-17]. Therefore, we chose to employ upper arm CVPs (UACVPs) rather than chest CVPs to prevent the possible complications associated with chest CVPs [18] and hypothesized that UACVPs could be one of the feasible options for CVPs, especially for systemic chemotherapy. In order to re-evaluate the utility of UACVPs, we examined UACVP-associated complications and long-term utility of UACVPs in a larger cohort of our patients.

\section{Patients and methods}

We reviewed the medical records of all patients who underwent subcutaneous implantation of UACVPs at Kyoto University Hospital from 1 April, 2006 to 30 June, 2009. UACVPs were implanted in 433 consecutive patients for the long-term administration of chemotherapy or fluid supplementation during this period, according to the implantation techniques described previously [18]. Almost all the patients $(427,98.6 \%)$ suffered from malignant diseases including colon cancer $(235,54.3 \%)$, gastric cancer $(45,10.4 \%)$, breast cancer $(45,10.4 \%)$, lung cancer $(29,6.7 \%)$, pancreatic cancer $(19,4.4 \%)$, esophageal cancer $(18,4.2 \%)$ and other malignancies $(36,8.3 \%)$. The majority of these patients $(386,89.1 \%)$ required the implantation of UACVPs for systemic CTx (CTx group). For the remaining 47 patients, the UACVPs were implanted for fluid supplementation, or because of inaccessibility to the peripheral blood vessels (non-CTx group). Furthermore, 38 patients from the non-CTx group were at the end stage of their malignancies, and required best supportive care. We performed routine computed tomography scanning every 3 or 4 months in all patients
Fig. 1 Subcutaneously implanted UACVP and representative manifestations of UACVP-related complications. a Titanium Vital Port system. b, c CVP was implanted on the ulnar side in the upper arm. d Port pocket infection with erythematic induration. e Skin dehiscence leading to the exposure of a subcutaneous CVP. $\mathbf{f}$ Venous thrombosis resulting in swelling of the left upper extremity. The patient underwent systemic anticoagulant therapy via the right peripheral blood vessel. g Bilateral pulmonary embolism. $\mathbf{h}$ Thrombus detected around the catheter in the subclavian vein. $\mathbf{i}, \mathbf{j}$ Catheter dislocation on threedimensional computed tomography

from the CTx group to assess the effectiveness of the treatment, and to detect UACVP-related complications including venous thrombosis. Using the same implantation protocol guided by ultrasonography (US) [18], all procedures were performed by senior surgeons under local anesthesia in a day surgery unit equipped with a mobile X-ray fluoroscopic scanner and US device. The UACVPs were implanted in the patient's non-dominant upper arm, with the exception of breast cancer patients with axillary lymph node dissection, who underwent UACVP implantation in the unaffected upper arm. In 2006, we employed a SlimPort ${ }^{\circledR}$ system (Bard Access Systems, Salt Lake City, UT, USA), but later decided to employ a Titanium Vital Port ${ }^{\circledR}$ system (Cook Vascular, Leechburg, PA, USA)(Fig. 1a) since the latter port is smaller and seems to be more suitable for subcutaneous implantation in the upper arm. We followed the patients with implanted UACVPs until March 2014. Follow-up was discontinued upon removal of the UACVPs, at the patient's death, or at the end of the study period. The information was retrieved from the medical charts, and the data included UACVP-related infections, i.e., catheter infection and port pocket infection (Fig. 1d)], skin dehiscence (Fig. 1e), venous thrombosis (Fig. 1f-h), catheter dislocation (Fig. 1i, j) or occlusion, port chamber twist, port leakage, catheter fracture and needle dislodgement. Catheter infection was defined by the following conditions-(1) when blood culture tests were positive for microorganisms including a coagulasenegative staphylococcus (a typical pathogen associated with long-term indwelling of venous catheters), or (2) when blood culture tests were negative but a high fever persisted without any possible infection foci other than the CVPs. Port pocket infection was defined as an erythematic or painful induration, or tenderness at the port site often complicated with pus retention. Venous thrombosis was confirmed by enhanced computed tomography, magnetic resonance imaging or US. Catheter occlusion was defined as the failure to aspirate and flush the contents via CVPs. In this study, aspiration occlusion, where the blood cannot be aspirated but the port/catheter system can be flushed without resistance, was not regarded as catheter occlusion, since aspiration occlusion can be episodic and normal catheter function can recover spontaneously without 

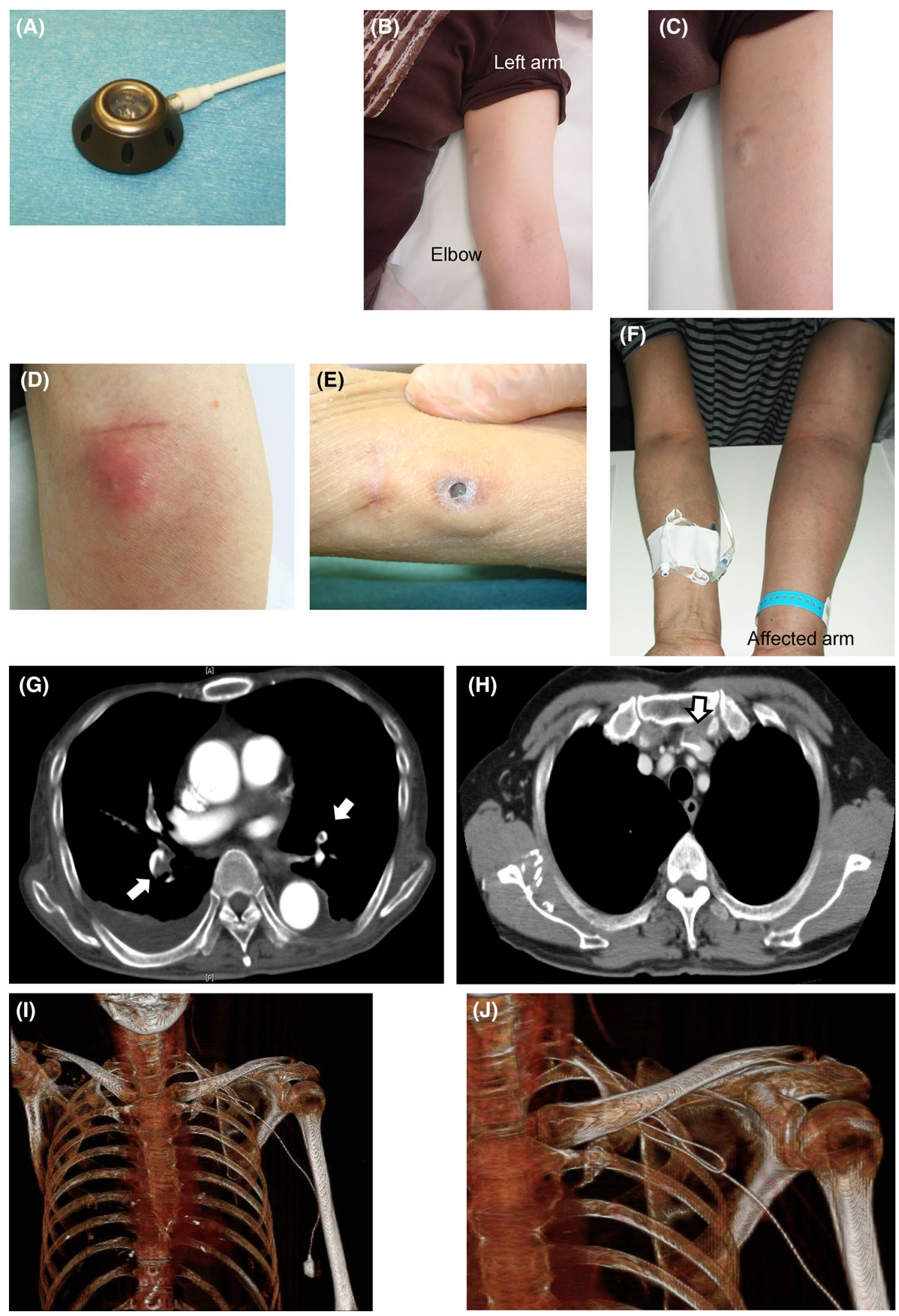
any intervention [18]. The study was conducted in accordance with the Helsinki Declaration, and the protocol was approved by the Ethics Committee of Kyoto University Hospital (Approval No. E-845).

\section{Statistical analysis}

The time to the development of UACVP-related complications and to port removal, i.e., complication-free and overall (removal-free) port availability, respectively was

Table 1 Patient background

\begin{tabular}{ll}
\hline Characteristics & \\
\hline Total $(n)$ & 433 \\
Patient gender & \\
Female & 186 \\
Male & 247 \\
Age (years) (Mean $\pm \mathrm{SD})$ & $63.39 \pm 11.0$ \\
Primary disease & \\
Malignancy & 427 \\
Benign & 6 \\
Purpose of the implant & \\
Chemotherapy & 386 \\
Other & 47 \\
Median duration of implant (range days) & $439.0(1-2824)$ \\
Total duration of implant (days) & 251,538 \\
\hline
\end{tabular}

evaluated using the Kaplan-Meier product limit method. Patient death and port removal without any UACVP-associated complications, including scheduled removal at the cessation of chemotherapy, were analyzed as censored cases. Differences in UACVP-related complication rates by the addition of bevacizumab (Bmab) were evaluated using Fisher's exact test. Statistical significance was assumed to be $p<0.05$. All statistical analyses were performed using JMP version 9.0.0 (SAS Institute Inc., USA).

\section{Results}

A total of 433 ports were implanted within five attempts without any complications including pneumothorax, hemothorax, arterial puncture, or cardiovascular problems. The median indwelling period was 439.0 days (1-2824), the cumulative follow-up period was 251,538 catheter days, and the median event free period was 422.0 days (1-2824) (Table 1). The median indwelling periods were 499.0 (1-2824) and 22.0 (1-2047) days in the CTx and non-CTx groups, respectively. In $87.2 \%$ (41/47) of patients in the non-CTx group, the indwelling periods were $<6$ months. In contrast, $60.6 \%$ (234/386) of patients in the CTx group could use UACVP for more than a year (Fig. 2). In the CTx group, $74(19.2 \%)$ patients received systemic CTx including Bmab, a recombinant humanized monoclonal antibody directed against vascular endothelial growth factor, and
Fig. 2 Indwelling periods of UACVPs according to the purpose of implantation. The black and gray bars represent the number of patients in the CTx and non-CTx groups, respectively. The median indwelling period was much shorter in the non-CTx group [22.0 (1-2047) days] compared to the CTx group [499.0 (1-2824) days]

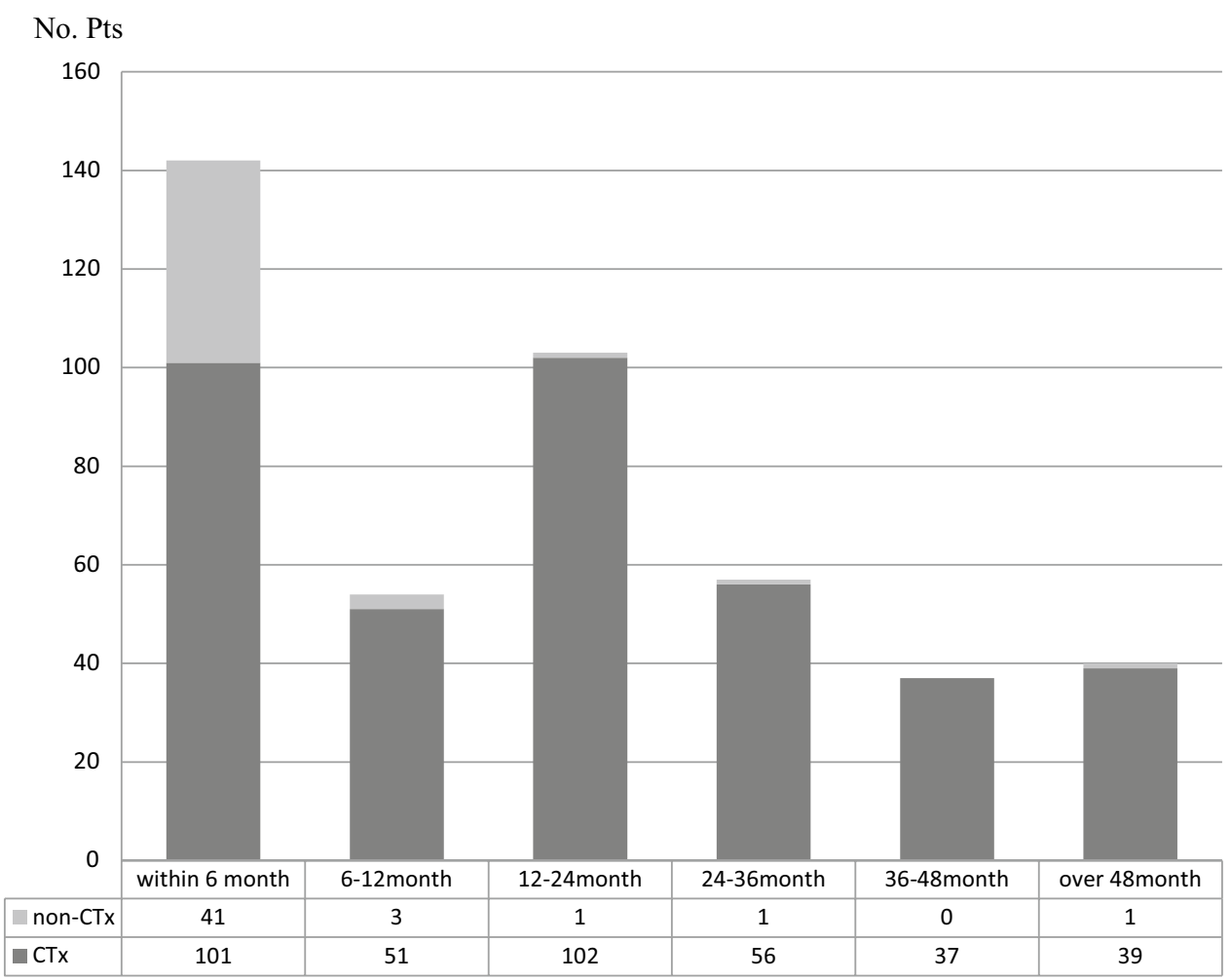


Fig. 3 Complication-free 1 -year port availability (solid line) and the overall one-year port availability (dotted line) in all patients were evaluated by the Kaplan-Meier method. The complication-free 1-year port availability was estimated at $86.0 \%$, whereas the overall 1 -year port availability in the whole group was estimated at $87.7 \%$

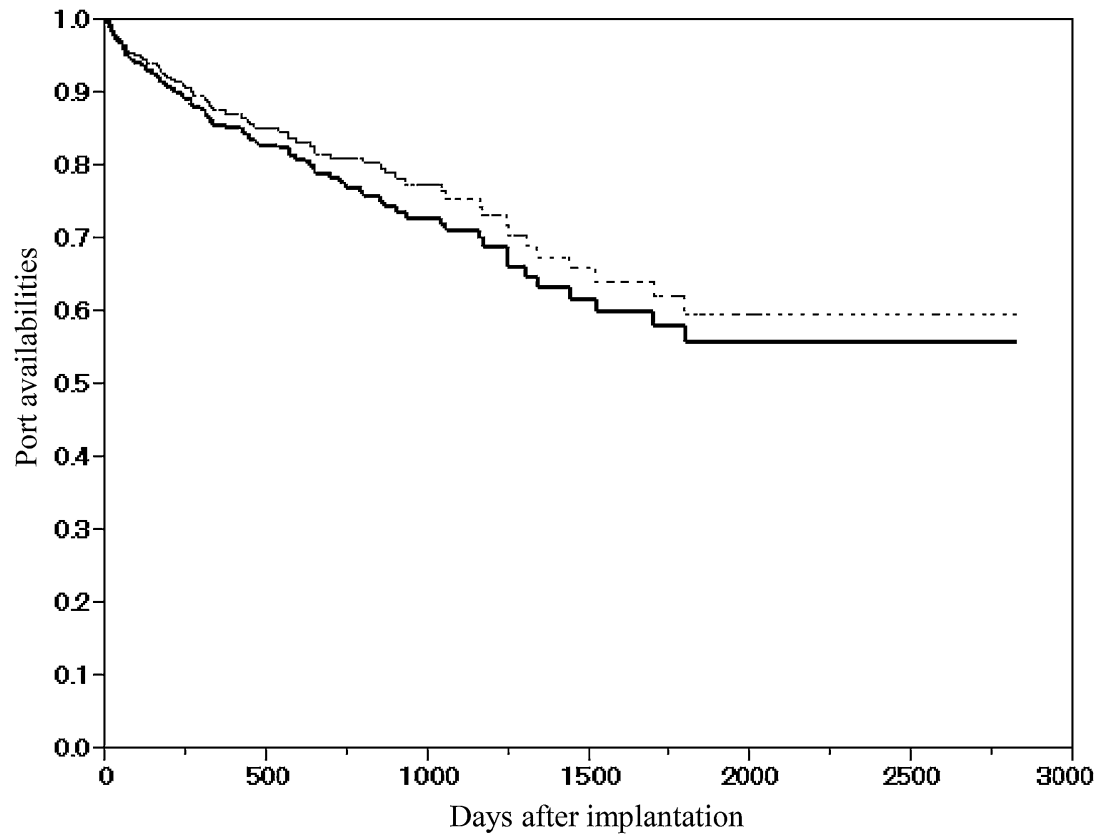

Table 2 CVP-related complications

\begin{tabular}{|c|c|c|c|c|c|c|}
\hline \multirow[t]{2}{*}{ Complications } & \multirow[t]{2}{*}{$n$} & \multirow{2}{*}{$\begin{array}{l}\text { Complication/ } 1000 \text { catheter days } \\
\text { (complication rate; \%) }\end{array}$} & \multirow[t]{2}{*}{ Evulsion } & \multicolumn{3}{|c|}{ Treatment for the patients without evulsion } \\
\hline & & & & Antibiotic & Anticoagulant & Observation \\
\hline Catheter infection & 27 & $0.107(6.2)$ & 27 & 0 & 0 & 0 \\
\hline Port infection & 16 & $0.064(3.7)$ & 14 & 2 & 0 & 0 \\
\hline Thrombosis & 10 & $0.040(2.3)$ & 3 & 0 & 7 & 0 \\
\hline Obstruction & 10 & $0.040(2.3)$ & 9 & 0 & 0 & 1 \\
\hline Catheter dislocation & 9 & $0.036(2.1)$ & 9 & 0 & 0 & 0 \\
\hline Reserver leak & 5 & $0.020(1.2)$ & 4 & 0 & 0 & 1 \\
\hline Skin complication (exposure) & 4 & $0.016(1.0)$ & 4 & 0 & 0 & 0 \\
\hline Port rotation/flip & 2 & $0.008(0.5)$ & 2 & 0 & 0 & 0 \\
\hline Total & 83 & & 72 & 2 & 7 & 2 \\
\hline
\end{tabular}

the cumulative follow-up period and the median indwelling periods were 77,141 catheter days and 983.0 days (85-2810), respectively. The complication-free one-year port availability in all groups and the CTx group only was estimated at 86.0 and $87.3 \%$, respectively, whereas the overall one-year port availability in all groups and the CTx group only was estimated at 87.7 and $88.7 \%$, respectively (Fig. 3).

There was no UACVP-related mortality throughout this study. Of the 142 patients with an indwelling period $<6$ months, 47 out of 101 patients in the CTx group and 31 out of 41 patients in the non-CTx group died due to deterioration of their primary disease without any UACVPrelated complications. During the long-term follow-up, UACVP-related complications occurred in a total of 83 patients (19.2\%) (Table 2). UACVP-related infections were evident in 43 patients (9.9\%, 0.17/1000 catheter days), and
27 of those patients were diagnosed with catheter infection (6.2\%, 0.11/1000 catheter days). A blood culture test was positive for bacteria in seven patients (1.6\%, 0.03/1000 catheter days) and for fungi in four patients $(0.9 \%$, $0.02 / 1000$ catheter days), but was negative in 12 patients. Of these 12 negative patients, a culture test for the catheter was also negative in 10 patients, but had not been performed in the remaining two patients. The UACVPs were removed in all patients suspected of catheter infection, who all recovered following the appropriate anti-bacterial and/ or anti-fungal treatment. Port pocket infection occurred in 16 patients $(3.7 \%, 0.064 / 1000$ catheter days), and was treated by port removal in ten patients and by administration of broad-spectrum antibiotics in the other two patients. In 44 patients from the non-CTx group for fluid supplementation, catheter and port pocket infections were evident in $3(6.8 \%)$ and $2(4.5 \%)$ patients, respectively. In contrast, 
Fig. 4 Indwelling periods at the onset of UACVP-related complications. Among the 74 patients with indwelling periods $>3$ years, 13 developed UACVP-related complications. UACVP-related infection tended to occur more frequently within one year after UACVP implantation, whereas venous thrombosis and catheter occlusion occurred regardless of the indwelling period. The parentheses in the bottom line indicate the number of patients with complications with UACVPs during the indicated period

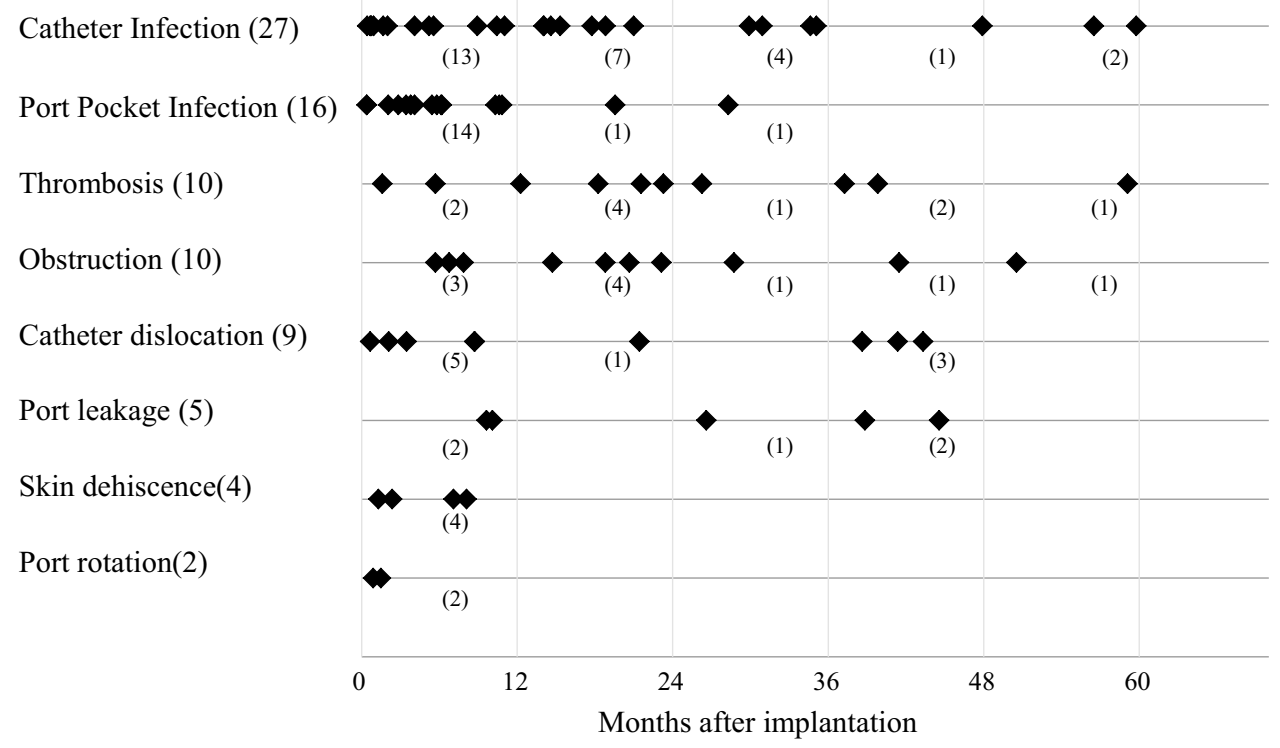

in the 389 patients from the CTx group, 24 (6.2\%) and 14 $(3.6 \%)$ patients developed catheter and port pocket infections, respectively. There were no significant differences in infection rates (catheter and port pocket) between the nonCTx and CTx groups $(p=0.80)$.

Of the ten patients with venous thrombosis $(2.3 \%$, $0.04 / 1000$ catheter days), three complained of thrombosis-related symptoms (swelling of the upper extremities, shoulder pain and respiratory distress), and systemic anticoagulant therapy was initiated promptly with a gradual amelioration of their symptoms. Asymptomatic thrombosis was detected in five patients on a regular computed tomography examination performed every 3 or 4 months. In addition, concomitant, asymptomatic pulmonary embolism was confirmed radiographically in two of these five patients. All thrombotic patients underwent systemic anticoagulant therapy, and following the disappearance of the thrombi by the appropriate therapy, the UACVPs were removed in three patients who had no need for further chemotherapy. Of the 74 patients receiving Bmab-containing CTx, UACVPrelated complications occurred in 22 patients (28.9\%, $0.29 / 1000$ catheter days), including nine cases of UACVPrelated infections (seven catheter infection and two port pocket infection) (12.2 \%, 0.12/1000 catheter days), three cases of venous thrombosis $(4.1 \%, 0.04 / 1000$ catheter days $)$ and four cases of catheter occlusion $(5.4 \%, 0.052 / 1000$ catheter days). Although the frequency of CVP-related complications tended to be higher in the Bmab-containing CTx group compared to the Bmab-free group $(p=0.022)$, there were no significant differences in the incidences of venous thrombosis between the two groups $(p=0.4119)$.

Of the nine patients with catheter dislocations $(2.1 \%$, $0.036 / 1000$ catheter days), two complained of chest or back pain at the initiation of routine CTx, whereas the remaining seven patients were asymptomatic, and the catheter dislocation was detected by a regular computed tomography examination. Port leakage was evident in five patients- the system was removed in four patients, and the other patient complained of mild swelling at the port pocket after each administration of CTx, possibly due to minimal leakage of the infusion. Since the patient's performance status was unsatisfactory and there were no signs of infection, we did not replace it with a new system, and the cause of the port leakage remained unclear in this case. There were no cases of detectable fibrin sheath formation, catheter fracture or needle dislodgement. In a total of 118 patients including 47 patients whose UACVPs were removed due to completion of chemotherapy, 31 (26.3\%) developed catheter infection, which was the main cause leading to port removal due to complications (Table 2).

With regard to the indwelling period at the onset of the UACVP-related complications (Fig. 4), port pocket infection, skin dehiscence and port leakage occurred within 1 year after UACVP implantation. Although half of catheter infection cases $(13 / 27,48 \%)$ were evident within 1 year after UACVP implantation, seven patients developed catheter infection $>2$ years after implantation. In contrast, venous thrombosis and catheter occlusion occurred regardless of the indwelling period.

\section{Discussion}

Although there are some arguments regarding the implantation site for CVPs, we have adopted UACVPs rather than chest CVPs because of the easier and safer access to the 
central blood vessel, as supported by Marcy et al. [16] In this study, the UACVPs were placed successfully in all patients without any implantation-related complications, including pneumothorax, hemothorax, arterial puncture, or cardiovascular problems. The rates of UACVP-related complications including infection, thrombosis, dislocation and occlusion in this study were comparable to those of chest and forearm CVPs and other UACVPs reported previously [6, 7, 19-27] (Table 3). Consistent with a previous report describing US-guided implantation and the feasibility of UACVPs [18], we confirmed the safety of the implantation procedures and the long-term utility of UACVPs in a larger cohort of patients.

The incidence of CVP-related infection (catheter infection and port pocket infection) was low at $0.17 / 1000$ catheter days $(10.0 \%)$, although the routine administration of prophylactic antibiotics was not standard in this study. Since the proper usage of antibiotics in the management of CVPs has not been proposed yet, further prospective randomized studies are warranted to determine whether prophylactic antibiotics can effectively reduce the incidence of CVP-related infection. Regarding the management of CVP-related infections, the port system should be removed in cases of suspected bacteremia or confirmed bacteremia/ fungemia, along with the prompt commencement of proper antibacterial and/or antifungal treatment [28]. In cases of port pocket infection without any signs of bacteremia, port removal is not necessary for improvement of the infection, which can be treated by antibiotic therapy as in 2 out of 12 cases of port pocket infection in this study.

With regard to the incidence of venous thrombosis, it was speculated that the longer intravascular catheter of an arm CVP system could increase the risk of venous thrombosis [29]. However, Marcy et al. argued that catheterrelated venous thrombosis was not associated with catheter length [30, 31]. In accordance with their argument, an extensive analysis of forearm CVPs in a large cohort of patient's [27] as well as in our study actually demonstrated a lower incidence of venous thrombosis (Table 3). Regardless of the indwelling site of the CVPs, the prophylactic administration of anticoagulants to prevent venous thrombosis is not recommended according to the Standards, Options and Recommendations (SOR) [32] and other guidelines [33]. However, it is well known that cancer patients are predisposed to thromboembolic diseases, and that chemotherapy can also raise the risk of thrombosis $[34,35]$. In this study, asymptomatic venous thrombosis was detected in 7 out of the 10 patients, thereby preventing future life-threatening thrombosis. Therefore, one should pay attention to the development of asymptomatic thrombosis on regular computed tomography examinations in patients with UACVPs, regardless of the indwelling length (Fig. 3). In addition, although Doppler US is superior to

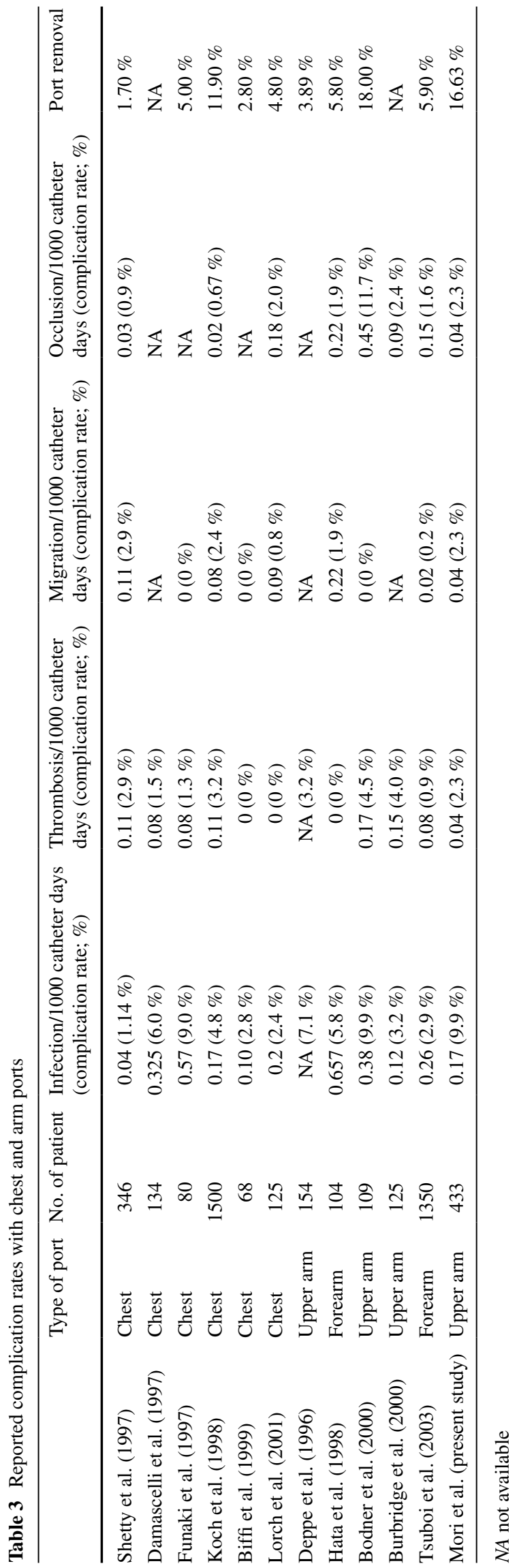


computed tomography in detecting venous thrombosis [36], we did not employ Doppler US in routine practice for the detection of venous thrombosis during the study period. Therefore, there was a possibility of underestimating the incidences of catheter-related thrombosis.

We examined whether the Bmab-containing CTx group had a tendency to develop venous thrombosis or not. According to a large pooled analysis of 6,055 cancer patients, the addition of Bmab to chemotherapy did not statistically significantly increase the risk of venous thromboembolisms compared to chemotherapy alone [37]. In our study, no significant difference was observed in the incidences of venous thrombosis between the Bmab-containing CTx group and the Bmab-free group $(p=0.4119)$. However the frequency of CVP-related complications tended to be higher in the Bmab-containing CTx group compared to the Bmab-free group $(p=0.022)$. Since the usage of CVPs was not evaluated in the pooled analysis, additional investigation is required to evaluate the effects of $\mathrm{Bmab}$ on the incidence of venous thrombosis and CVP-related complications.

The incidence of catheter dislocation in our study $(2.1 \%, 0.036 / 1000$ catheter days) was compatible to that of previous reports [23-27]. Although we could not clarify the mechanisms responsible for the dislocation, extreme motion of the upper extremities might induce a migration of the catheter tip into the innominate or subclavian veins. Since a catheter dislocating into narrow veins can cause venous thrombosis, the catheter should be removed even when patients remain asymptomatic. Furthermore, if the patient complains of chest or back pain at the initiation of systemic administration or if catheter dysfunction occurs, the tip position of the catheter should be immediately evaluated by chest radiography or fluoroscopy with contrast medium. These precautions can prevent serious complications due to catheter dislocation. In ours study, no serious complications associated with catheter dislocation have occurred so far.

In our study, there were four cases of skin dehiscence and two cases of port chamber twist. In order to minimize skin dehiscence, it seemed important to implant the UACVPs under the subcutaneous fat tissue, not just under the dermis. In one case of port chamber twist, the port was not fixed with any sutures. In the other case, the reason why the port was inverted in the small pocket remained unresolved. It would be desirable to fix the port with a few sutures in a pocket which just fits the size of that port.

UACVPs are well accepted, especially by female patients, in terms of more convenient CTx since they do not have to get undressed for the insertion or removal of a needle, thereby relieving the embarrassment of undressing. In addition, UACVPs can be easily covered with a shortsleeved shirt. Since UACVPs are generally smaller than chest CVPs, one can experience some difficulty in inserting the needle into a smaller puncture area of the UACVPs, especially in obese patients. The self-insertion of a needle is also sometimes difficult, since patients with UACVPs cannot hold the CVPs by themselves when they insert the needle. Therefore, UACVPs seem unsuitable for long-term total parenteral nutrition in patients with short-bowel syndrome and other gastrointestinal disorders, although the short-term usage of UACVPs can be useful for fluid supplementation in advanced cancer patients as in the nonCTx group.

In conclusion, UACVPs seem to be feasible especially for systemic CTx in terms of port availability, and could be one of the options for CVP implantation in patients with some difficulties in accessing their subclavian or internal jugular veins. However, since this is a retrospective study with some limitations, a randomized clinical trial comparing the safety and utility between UACVPs and chest CVPs (including internal jugular vein access) is warranted.

\section{Compliance with ethical standards}

Conflict of interest The authors declare that they have no conflict of interest.

Open Access This article is distributed under the terms of the Creative Commons Attribution 4.0 International License (http://creativecommons.org/licenses/by/4.0/), which permits unrestricted use, distribution, and reproduction in any medium, provided you give appropriate credit to the original author(s) and the source, provide a link to the Creative Commons license, and indicate if changes were made.

\section{References}

1. Engstrom PF, Arnoletti JP, Benson AB 3rd et al (2009) NCCN Clinical practice guidelines in oncology: colon cancer. J Natl Compr Canc Netw 7:778-831

2. Engstrom PF, Arnoletti JP, Benson AB 3rd et al (2009) NCCN Clinical Practice Guidelines in Oncology: rectal cancer. J Natl Compr Canc Netw 7:838-881

3. Benson AB 3rd, Bekaii-Saab T, Chan E et al (2013) Metastatic colon cancer, version 3.2013: featured updates to the NCCN Guidelines. J Natl Compr Canc Netw 11(2):141-152

4. Conroy T, Desseigne F, Ychou M et al (2011) FOLFIRINOX versus gemcitabine for metastatic pancreatic cancer. N Engl J Med 364(19):1817-1825

5. Di Carlo I, Cordio S, La Greca G et al (2001) Totally implantable venous access devices implanted surgically: a retrospective study on early and late complications. Arch Surg 136:1050-1053

6. Lorch H, Zwaan M, Kagel C et al (2001) Central venous access ports placed by interventional radiologists: experience with 125 consecutive patients. Cardiovasc Intervent Radiol 24:180-184

7. Damascelli B, Patelli G, Frigerio LF et al (1997) Placement of long-term central venous catheters in outpatients: study of 134 patients over 24596 catheter-days. Am J Roentgenol 168:1235-1239 
8. Sakamoto N, Arai Y, Takeuchi Y et al (2010) Ultrasound-guided radiological placement of central venous port via the subclavian vein: a retrospective analysis of 500 cases at a single institute. Cardiovasc Intervent Radiol 33:989-994

9. Hinke DH, Zandt-Stastny DA, Goodman LR et al (1990) Pinchoff syndrome: a complication of implantable subclavian venous access devices. Radiology 177:353-356

10. Klotz HP, Schöpke W, Kohler A et al (1996) Catheter fracture: a rare complication of totally implantable subclavian venous access devices. J Surg Oncol 62:222-225

11. Mirza B, Vanek VW, Kupensky DT (2004) Pinch-off syndrome: case report and collective review of the literature. Am Surg 70:635-644

12. Pittiruti M, Malerba M, Carriero C et al (2000) Which is the easiest and safest technique for central venous access? A retrospective survey of more than 5400 cases. J Vasc Access 1:100-107

13. Schuman E, Brady A, Gross G et al (1987) Vascular access options for outpatient cancer therapy. Am J Surg 153:487-489

14. Teichgräber UK, Kausche S, Nagel SN et al (2011) Outcome analysis in 3160 implantations of radiologically guided placements of totally implantable central venous port systems. Eur Radiol 21:1224-1232

15. Sofocleous CT, Schur I, Cooper SG et al (1998) Sonographically guided placement of peripherally inserted central venous catheters: review of 355 procedures. Am J Roentgenol 170:1613-1616

16. Marcy PY, Figl A, Amoretti N et al (2010) Arm port implantation in cancer patients. Int J Clin Oncol 15:328-330

17. Marcy PY, Magné N, Castadot $P$ et al (2007) Is radiologic placement of an arm port mandatory in oncology patients: analysis of a large bi-institutional experience. Cancer 110:2331-2338

18. Kawamura J, Nagayama S, Nomura A et al (2008) Long-term outcomes of peripheral arm ports implanted in patients with colorectal cancer. Int J Clin Oncol 13:349-354

19. Shetty PC, Mody MK, Kastan DJ et al (1997) Outcome of 350 implanted chest ports placed by interventional radiologists. J Vasc Interv Radiol 8:991-995

20. Funaki B, Szymski GX, Hackworth CA et al (1997) Radiologic placement of subcutaneous infusion chest ports for long-time central venous access. Am J Roentgenol 169:1431-1434

21. Koch HJ, Pietsch M, Krause U et al (1998) Implantable vascular access systems: experience in 1500 patients with totally implanted central venous port systems. World J Surg 22:12-16

22. Biffi R, Martinelli G, Pozzi S et al (1999) Totally implantable central venous access ports for high-dose chemotherapy administration and autologous stem cell transplantation: analysis of overall and septic complications in 68 cases using a single type of device. Bone Marrow Transplant 24:89-93

23. Deppe G, Kahn ML, Malviya VK et al (1996) Experience with the P.A.S.-PORT venous access device in patients with gynecologic malignancies. Gynecol Oncol 62:340-343
24. Hata Y, Morita S, Morita Y et al (1998) Peripheral insertion of a central venous access device under fl uoroscopic guidance using a peripherally accessed system (PAS) port in the forearm. Cardiovasc Intervent Radiol 21:230-233

25. Bodner LJ, Nosher JL, Patel KM et al (2000) Peripheral venous access ports: outcomes analysis in 109 patients. Cardiovasc Intervent Radiol 23:187-193

26. Burbridge B, Krieger E, Stoneham G (2000) Arm placement of the cook titanium petite vital-port: results of radiologic placement in 125 patients with cancer. Can Assoc Radiol J 51:163-169

27. Tsuboi N, Morita S, Yamanishi T et al (2003) Long-Term outcomes of a totally implantable central venous access system in the forearm. Jpn J Intervent Radiol 18:43-48

28. Vescia S, Baumagartner AK, Jacobs VR et al (2008) Management of venous port systems in oncology: a review of current evidence. Ann Oncol 19:9-15

29. Kuriakose P, Colon-Otero G, Paz-Fumagalli R (2002) Risk of deep venous thrombosis associated with chest versus arm central venous subcutaneous port catheters: a 5 years single-institution retrospective study. J Vasc Interv Radiol 13:179-184

30. Marcy PY, Chamorey E, Amoretti N et al (2008) A comparison between distal and proximal port device insertion in head and neck cancer. Eur J Surg Oncol 34:1262-1269

31. Marcy PY, Magné N, Castadot P et al (2005) Radiological and surgical placement of port devices: a 4 years institutional analysis of procedure performance, quality of life and cost in breast cancer patients. Breast Cancer Res Treat 92:61-67

32. Debourdeau P, Kassab Chahmi D, Le Gal G et al (2008) SOR guidelines for the prevention and treatment of thrombosis associated with central venous catheters in patients with cancer: report from the working group. Ann Oncol 2009(20):1459-1471

33. Khorana AA, Streiff MB, Farge D et al (2009) Venous thromboembolism prophylaxis and treatment in cancer: a consensus statement of major guidelines panels and call to action. J Clin Oncol 27:4919-4926

34. Heit JA, Silverstein MD, Mohr DN et al (2000) Risk factors for deep vein thrombosis and pulmonary embolism: a populationbased case-control study. Arch Intern Med 160:809-815

35. Silverstein MD, Heit JA, Mohr DN et al (1998) Trends in the incidence of deep vein thrombosis and pulmonary embolism: a 25 years population-based study. Arch Intern Med 158:585-593

36. Luciani A, Clement O, Halimi P et al (2001) Catheter-related upper extremity deep venous thrombosis in cancer patients: a prospective study based on Doppler US. Radiology 220:655-660

37. Hurwitz HI, Saltz LB, Cutsem EV et al (2011) Venous thromboembolic events with chemotherapy plus bevacizumab: a pooled analysis of patients in randomized phase II and III studies. J Clin Oncol 29:1757-1764 